\title{
Fonction osseuse : fos et les autres
}

L'os est un tissu dynamique. Le remodelage osseux, c'est-à-dire la résorption de l'os minéralisé suivie de l'ostéogenèse d'os nouveau, est un phénomène complexe faisant intervenir deux types cellulaires distincts, l'ostéoblaste et l'ostéoclaste, dont les fonctions sont modulées par de nombreuses cytokines et hormones. Des maladies sévères, telles l'ostéoporose, l'ostéopétrose ou la maladie de Paget, surviennent lorsque l'équilibre entre ostéogenèse et résorption osseuse est perturbé. Bien que les bases génétiques ou moléculaires à l'origine de ces maladies soient diverses, les résultats spectaculaires de travaux récents [1-3] démontrent l'implication de certains proto-oncogènes, $c$-fos et $c$-src, dans la fonction osseuse, l'activité ostéoclastique et l'étiologie de l'ostéopétrose. Les proto-oncogènes sont des gènes cellulaires dont la forme activée, appelée oncogène, est impliquée dans la carcinogenèse. Il est maintenant bien établi que les protooncogènes jouent un rôle important dans le contrôle de la croissance et de la différenciation cellulaires. Le proto-oncogène $c$-fos est le gène cellulaire correspondant à l'oncogène activé v-fos identifié à l'origine comme étant l'oncogène transformant des virus de sarcomes murins FBJ et FBR $[4,5]$. La protéine Fos est une phosphoprotéine nucléaire formant un complexe stable avec le produit d'un autre proto-oncogène récemment identifié, c-jun [6]. Ces deux gènes sont les prototypes de deux familles de gènes qui comprennent, d'une part, $c$-fos, fos- $B$, fra-1 et fra-2, et d'autre part c-jun, jun-B et jun-D [4].

D'élégantes études biochimiques ont révélé les détails de l'interaction structurelle et fonctionnelle entre Fos et Jun. Les différents membres de la famille fos forment des hétérodimères avec les membres de la famille jun à travers un motif pro$\mathrm{m} / \mathrm{s} n^{\circ} 11$ vol. 9, novembre 93 téique appelé glissière à leucines (leucine zipper), qui consiste en une série de résidus leucines espacés de six résidus d'acides aminés et alignés sur la face externe d'une région formant une hélice $\alpha$ [7]. Les deux hélices $\alpha$ de deux glissières à leucines semblent s'imbriquer l'une dans l'autre dans une orientation parallèle grâce aux interactions hydrophobes entre les résidus leucines qui stabilisent ainsi la formation du dimère. L'hétérodimère ainsi formé constitue la forme active de la molécule qui peut se lier à l'ADN sur des sites spécifiques nommés sites AP-1, et agir comme facteur de transcription pour contrôler l'expression de gènes dont les promoteurs contiennent des sites AP-1 [8]. Les différents membres de la famille jun peuvent aussi former des homo- ou des hétérodimères entre eux et remplir les mêmes fonctions (figure 1) [9].

D'innombrables études ont examiné l'expression de c-fos dans plusieurs tissus et types cellulaires en culture. Le traitement de tissus, ou de cellules, avec différents agonistes stimule de façon importante le niveau d'ARNm de c-fos [10]. En particulier, l'expression de $c$-fos est induite lorsque des cellules quiescentes réintègrent le cycle cellulaire, ce qui a amené plusieurs laboratoires à proposer un rôle essentiel pour c-fos dans le cycle et la croissance cellulaires. Cependant, un certain nombre de travaux suggèrent plutôt un rôle précis de $c$-fos dans la physiologie de certains tissus particuliers, tels l'os et le cerveau. En effet, l'expression de c-fos est induite dans différentes régions du cerveau par la stimulation électrique, la stimulation sensorielle, ou par des substances provoquant des convulsions épileptiques [11]. Dans l'os, c-fos est exprimé au cours de l'embryogenèse $[12,13]$, de même que dans des ostéoblastes en culture traités par l'hormone de croissance, les facteurs de croissance proches de l'insuline (insulin-like growth factors IGF-I et IGF-II), et par la forme active de la vitamine D [14-16]. Des souris transgéniques exprimant des niveaux élevés de $c$-fos développent des anomalies du développement des os longs [17], et la forme activée de l'oncogène, v-fos, provoque des ostéosarcomes [5]. Tous ces travaux suggèrent une implication de c-fos dans la physiologie osseuse. Les travaux récents de deux groupes ont confirmé cette hypothèse de façon spectaculaire.

Les laboratoires du Dr Bruce Spiegelman (Boston, MA, USA) et du Dr Erwin Wagner (Vienne, Autriche) ont, indépendamment, créé des lignées de souris transgéniques déficientes en $c$-fos $[1,2]$. L'inactivation du locus c-fos a été réalisée par recombinaison homologue dans des cellules souches embryonnaires (ES) de souris $[18,19]$. Ces cellules, maintenues dans un phénotype non différencié in vitro, peuvent être facilement transfectées avec un vecteur contenant une mutation dans le gène ciblé. Le groupe de Spiegelman a inactivé le locus c-fos par intégration du gène de sélection neo dans le premier exon, alors que le groupe autrichien a choisi d'inactiver fos par insertion de la cassette neo dans le second exon du gène. De puissants protocoles de double sélection ont été mis au point afin de discriminer l'intégration au hasard du vecteur de son intégration spécifique au site visé, à la suite d'une recombinaison homologue [20]. Après sélection des clones de cellules ES ayant intégré l'allèle mutant au site chromosomique visé, les cellules sont injectées dans un blastocyste afin de former une chimère. Les blastocystes sont ensuite réimplantés chez des femelles pseudo-gestantes. De façon générale, des lignées de souris au pelage dif- 




Figure 1. Le facteur de transcription AP-1 (fos/jun). Un agoniste induit la transcription des différents membres des familles fos et jun à travers une cascade de communication des signaux intracellulaires. Les protéines Fos et Jun sont traduites et transposées au noyau où elles forment des homo- et des hétérodimères. Les membres de la famille Fos ne peuvent que former des hétérodimères avec les protéines Jun alors que celles-ci peuvent aussi former des homo- ou des hétérodimères entre elles. Les cylindres des protéines stylisées représentent les hélices $\alpha$ des glissières à leucines. Les dimères constituent la forme active de la molécule et forment le facteur de transcription AP-1 se liant à I'ADN au site illustré.

férent sont choisies afin d'isoler les cellules ES et les blastocystes. Il est donc aisé d'identifier les chimères ayant intégré les cellules ES puisqu'elles ont un pelage tacheté (par exemple agouti sur fond noir). Ces animaux sont ensuite accouplés afin d'identifier ceux dont les gamètes ont incorporé l'allèle mutant (cela est accompli par l'observation du pelage et la détermination du génotype). Les animaux hétérozygotes pour la mutation désirée sont finalement croisés entre eux afin d'obtenir des homozygotes. Les deux groupes ont réussi avec succès à éliminer l'expression de protéine Fos active, et le phénotype des homozygotes, obtenus indépendamment par les deux laboratoires, est remarquablement identique.

L'observation la plus surprenante faite à l'occasion de ces études est que les homozygotes sont viables, bien que de plus petite taille. Cela est incompatible avec l'hypothèse assignant un rôle central à $c$-fos dans le contrôle du cycle cellulaire. Les conclusions erronées des nombreux travaux, semblant démontrer le besoin essentiel de $c$-fos pour la progression à travers le cycle cellulaire, résultent sans doute d'artefacts liés aux techniques utilisées, l'usage de séquences fos anti-sens [21], ou l'injection d'anticorps dirigés contre
Fos [22]. Les réactifs employés n'étaient sans doute pas suffisamment spécifiques, et il est probable que les molécules utilisées inhibaient plusieurs membres de l'une ou l'autre des familles fos et jun, ce qui pourrait être plus dommageable pour le cycle cellulaire. En effet, des travaux récents, utilisant des anticorps dont la spécificité est exclusive de Fos, ont démontré que la protéine Fos peut être inactivée sans empêcher la cellule de croître [23]. Ces résultats ont été validés de façon convaincante par les souris mutantes homozygotes.

Les souris fos-/- ont une hématopoièse déficiente (lymphopénie), 
une gamétogenèse altérée ou absente, et un comportement anormal. De plus, leur développement osseux est perturbé et les animaux présentent un phénotype ostéopétrotique avec un raccourcissement des os longs, une ossification de l'espace médullaire, et l'absence de dents [1, 2]. Les cellules affectées sont donc principalement les cellules somatiques qui montrent une expression réglée de c-fos in vivo [11-16].

L'ostéopétrose se caractérise par un tissu osseux dense et un faible développement de la cavité médullaire des os contenant la moelle osseuse. Ce phénotype semble dû à une incapacité des ostéoclastes à résorber l'os minéralisé [23], bien que le nombre d'ostéoclastes présents dans le tissu ne semble pas affecté outre mesure [24]. Dans le cas des homozygotes fos -/-, l'activité des ostéoclastes n'a pas encore été évaluée, ce qui empêche d'affirmer sans équivoque que ce sont ces cellules qui sont en cause. En effet, l'activité et la différenciation des ostéoclastes étant sous l'influence des ostéoblastes [25], il est possible que les cellules affectées par le phénotype fos -/- soient les ostéoblastes. De nombreux agonistes affectant l'un ou l'autre aspect de la physiologie cellulaire induisent l'expression des proto-oncogènes nucléaires, tel c-fos [10]. La stimulation de c-fos représente donc l'aboutissement d'une ou de plusieurs voies de transmission des signaux intracellulaires. Un phénotype identique peut provenir de l'inactivation de gènes distincts, agissant en amont de c-fos dans la cascade de transmission des signaux. Cela semble être le cas pour $c$-src, un autre proto-oncogène codant pour une tyrosine kinase. L'activation de l'activité kinasique de $c$-src stimule l'expression de c-fos [26]. De même, l'inactivation de $c$ src par recombinaison homologue induit un phénotype ostéopétrotique chez les homozygotes src-/- [3]. Il est donc probable que l'activation de l'expression de $c$-fos dans l'os résulte d'une cascade de transmission des signaux impliquant $c$-src [27]. De plus, les homozygotes src-/- montrent une altération de l'activité de résorption des ostéoclastes, ce qui $\mathrm{m} / \mathrm{s} n^{\circ} 11$ vol. 9, novembre 93 suggère que ce sont ces mêmes cellules qui sont affectées chez le mutant fos -/.

En revanche, l'inactivation de $c$-src au niveau du cerveau n'entraîne aucune modification histologique ou du comportement $[3,28]$, ce qui est une surprise, compte tenu de l'expression de $c$-src dans ce tissu. Dans le cerveau, la voie de transmission des signaux conduisant à la stimulation de l'expression de $c$-fos ne semble pas impliquer $c$-src, mais pourrait impliquer $c-f y n$, une autre tyrosine kinase de la même famille. En effet, l'inactivation de $c$-fyn par recombinaison homologue affecte les capacités d'apprentissage des souris homozygotes de manière comparable à ce qui a été observé chez les homozygotes fos-/- ([25] ; B.M. Spiegelman, communication personnelle).

De nombreux éléments restent à déchiffrer afin de préciser sans équivoque le rôle de certains protooncogènes au cours de la différenciation et du développement. En revanche, les puissantes techniques de recombinaison homologue ont bel et bien démontré le potentiel qu'on leur prêtait au départ. Les travaux récents ont confirmé certaines hypothèses mais révélé de grandes surprises : c-fos n'est pas essentiel à la progression du cycle cellulaire, mais remplit plutôt un rôle important dans la fonction de certains tissus particuliers, tels l'os et le cerveau. L'étude détaillée du phénotype des mutants fos-/- permettra de définir le rôle de c-fos dans l'ostéogenèse et la voie de différenciation des ostéoclastes. De même, la production de mutants impliquant des gènes de la même famille, ou des gènes impliqués dans les mêmes voies de transmission des signaux, précisera le modèle du rôle physiologique de mise en jeu du facteur de transcription AP-1 (Fos/Jun)

\section{René Saint-Arnaud}

Unité de génétique, Hôpital Shriners pour enfants et département de chirurgie et centre de génétique humaine, Université McGill, Montréal, H3G lA6 Canada.

\section{RÉFÉRENCES}

1. Johnson RS, Spiegelman BM, Papaioannou V. Pleiotropic effects of a null mutation in the c-fos proto-oncogene. Cell 1992 ; 71 : 577-86.

2. Wang ZQ, Ovitt C, Grigoriadis AE, Möhle-Steinlein U, Rüther U, Wagner EF. Bone and haematopoietic defects in mice lacking c-fos. Nature $1992 ; 360$ : 741-5.

3. Soriano P, Montgomery C, Geske R, Bradley A. Targeted disruption of the $c$-src proto-oncogene leads to osteopetrosis in mice. Cell 1991 ; 64 : 693-702.

4. Blanchard JM. Le proto-oncogène $c$-fos: un "entremetteur» moléculaire. médecine/sciences $1992 ; 8$ : 455-70.

5. Curran T, Peters G, Van Beveren C, Teich NM, Verma IM. FBJ murine osteosarcoma virus: identification and molecular cloning of biologically active proviral DNA. J Virol 1982; 44 : 674-82.

6. Rauscher FJ III, Cohen DR, Curran T, Bos TJ, Vogt PK, Bohmann D, Tjian R, Franza BR Jr. Fos-associated protein p39 is the product of the jun proto-oncogene Science 1988; 240: 1010-6.

7. Landschulz WH, Johnson PF, McKnight SL. The leucine zipper: a hypothetical structure common to a new class of DNA binding proteins. Science 1988; 240 : 1759-64

8. Chiu R, Boyle WJ, Meek J, Smeal T, Hunter T, Karin M. The c-Fos protein interacts with c-Jun/AP-1 to stimulate transcription of AP-1 responsive genes. Cell $1988 ; 54: 541-52$.

9. Nakabeppu Y, Ryder K, Nathans D DNA binding activities of three murine Jun proteins : stimulation by Fos. Cell 1988 ; 55 : 907-15.

10. Angel $P$, Karin M. The role of Jun, Fos and the AP-1 complex in cell-proliferation and transformation. Biochim Biophys Acta $1991 ; 1072$ : 129-57.

11. Sheng M, Greenberg ME. The regulation and function of $c$-fos and other immediate early genes in the nervous system. Neuron $1990 ; 4$ : 477-85.

12. Dony C, Gruss P. Protooncogene c-fos expression in growth regions of fetal bone and mesodermal web tissue. Nature 1987 328: 711-4

13. De Togni P, Niman H, Raymond V, Sawchenko P, Verma IM. Detection of Fos protein during osteogenesis by monoclonal antibodies. Mol Cell Biol 1988; 8: 2251-6.

14. Slootweg MC, van Genesen ST, Otte AP, Duursma SA, Kruijer W. Activation of mouse osteoblast growth hormone receptor: $c$-fos oncogene expression independent of phosphoinositide breakdown and cyclic AMP. J Mol Endocrinol 1990; 4 : 265-74.

15. Merriman HL, LaTour D, Linkhart TA, Mohan S, Baylink DJ, Strong DD. Insulinlike growth factor-I and insulin-like growth factor-II induce $c$-fos in mouse osteoblastic cells. Calcif Tissue Int 1990; 46 : 258-62. 
16. Candeliere GA, Prud'homme J, StArnaud R. Differential stimulation of fos and jun family members by calcitriol in osteoblastic cells. Mol Endocrinol 1991 ; 5 : 1780-8.

17. Rüther U, Garber C, Komitowski D, Müller R, Wagner EF. Deregulated $c$-fos expression interferes with normal bone development in transgenic mice. Nature 1987 ; 325 : 412-6.

18. Babinet C. Les cellules souches embryonnaires de souris: une voie privilégiée de transformation génétique à l'échelle de l'animal. médecine/sciences 1992; 8: 268-75.

19. Capecchi MR. Altering the genome by homologous recombination. Science 1989 ; 244: 1288-92.

20. Mansour SL, Thomas KR, Capecchi MR. Disruption of the proto-oncogene int-2 in mouse embryo-derived stem cells : a general strategy for targeting mutations to non-selectable genes. Nature 1988; 336 : 348-52.

21. Holt JT, Gopal TV, Moulton AD, Nienhuis AW. Inducible production of c-fos antisense RNA inhibits 3T3 cell proliferation. Proc Natl Acad Sci USA 1986; 83 : 4794-8. 22. Riabowol KT, Vosatka RJ, Ziff EB, Lamb NJ, Feramisco JR. Microinjection of fos-specific antibodies blocks DNA synthesis in fibroblast cells. Mol Cell Biol 1988; 8: $1670-6$.

23. Kovary K, Bravo R.The jun and fos protein families are both required for cell cycle progression in fibroblasts. Mol Cell Biol 1991; 11 : 4466-72.

24. Shapiro F, Glimcher MJ, Holtrop ME. Human osteopetrosis. A histological, ultrastructural and biochemical study. I Bone Joint Surg 1980; 62A: 384-99.

25. Shapiro F, Dey LL, Anast C. Variable osteoclast appearance in human infantile osteopetrosis. Calcif Tissue Int 1988; 43 : 67-76.

26. McSheehy PMJ, Chambers TJ. Osteoblast-like cells in the presence of parathyroid hormone release soluble factor that stimulates osteoclastic bone resorption. Endocrinology 1986; 119: 1654-9.

27. Fujii M, Shalloway D, Verma IM. Gene regulation by tyrosine kinases: src protein activates various promoters, including $c$-fos. Mol Cell Biol 1989; 9 : 2493-9.

28. Grant SGN, O’Dell TJ, Karl KA, Stein PL, Soriano P, Kandel ER. Impaired longterm potentiation, spatial learning, and hippocampal development in fyn mutant mice. Science 1992; 258: 1903-10.

\section{TIRÉS A PART}

R. Saint Arnaud. 